\title{
Open-Domain Question-Answering for COVID-19 and Other Emergent Domains
}

\author{
Sharon Levy§, Kevin Moף, Wenhan Xiong§, William Yang Wang§ \\ §University of California, Santa Barbara \\ IPrinceton University \\ \{sharonlevy, xwhan, william\}@cs.ucsb.edu, kevinmo@princeton.edu
}

\begin{abstract}
Since late 2019, COVID-19 has quickly emerged as the newest biomedical domain, resulting in a surge of new information. As with other emergent domains, the discussion surrounding the topic has been rapidly changing, leading to the spread of misinformation. This has created the need for a public space for users to ask questions and receive credible, scientific answers. To fulfill this need, we turn to the task of open-domain question-answering, which we can use to efficiently find answers to free-text questions from a large set of documents. In this work, we present such a system for the emergent domain of COVID-19. Despite the small data size available, we are able to successfully train the system to retrieve answers from a large-scale corpus of published COVID-19 scientific papers. Furthermore, we incorporate effective re-ranking and questionanswering techniques, such as document diversity and multiple answer spans. Our opendomain question-answering system can further act as a model for the quick development of similar systems that can be adapted and modified for other developing emergent domains.
\end{abstract}

\section{Introduction}

With the rise of social media and other online sources, it is easy to access information from sites without third-party filtering (Allcott and Gentzkow, 2017). As such, it is important in today's society to create systems that can provide credible and reliable information to users. This is especially true in the context of emergent domains which, unlike more established sectors, may contain rapidly changing information. COVID-19 follows this pattern, with over 100,000 related articles published in 2020 and new research findings still frequently reported (Else, 2020).

However, the vast interest and exposure surrounding this topic have consequently generated a rise in misinformation (Kouzy et al., 2020; Medina Serrano et al., 2020). This can lead to lower compliance with various preventative measures such as social distancing, which in turn can continue the spread of the virus (Bridgman et al., 2020; Tasnim et al., 2020). A question-answering system that allows users to ask free-text questions with answers deriving from published articles and reliable scientific sources can help mitigate this spread of misinformation and inform the public at the same time.

The task of open-domain question-answering has risen in prominence in recent years (Chen et al., 2017; Yang et al., 2019; Xiong et al., 2021a). Systems have evolved from keyword-based approaches (Salton and McGill, 1986) to the utilization of neural networks with dense passage retrieval (Xiong et al., 2021b). Furthermore, largescale datasets have been used to train and test these systems, such as general knowledge datasets (Joshi et al., 2017; Nguyen et al., 2016) and domainspecific datasets $^{1}$ (Tsatsaronis et al., 2012). However, many of these systems are evaluated on these established datasets with abundant questions and clearly defined answers. In the case of an emergent domain system, this likely will not be available and the reduced data size can result in lower answer precision.

In this paper, we build an open-domain questionanswering system in the emergent domain of COVID-19. We aim to overcome a staple issue with emergent domain question-answering systems: lack of data. While several COVID-19-related datasets have been published since the beginning of the pandemic (Roberts et al., 2020; Tang et al., 2020), they are small in scale and cannot be used for training our models. We tackle the issue of data shortage by fine-tuning pre-trained biomedical language models with a small in-domain dataset. Though these models are not trained on COVID19 data, they allow our system to warm start with general biomedical terminology. Other COVID-

\footnotetext{
${ }^{1}$ https://trec.nist.gov/data.html
} 


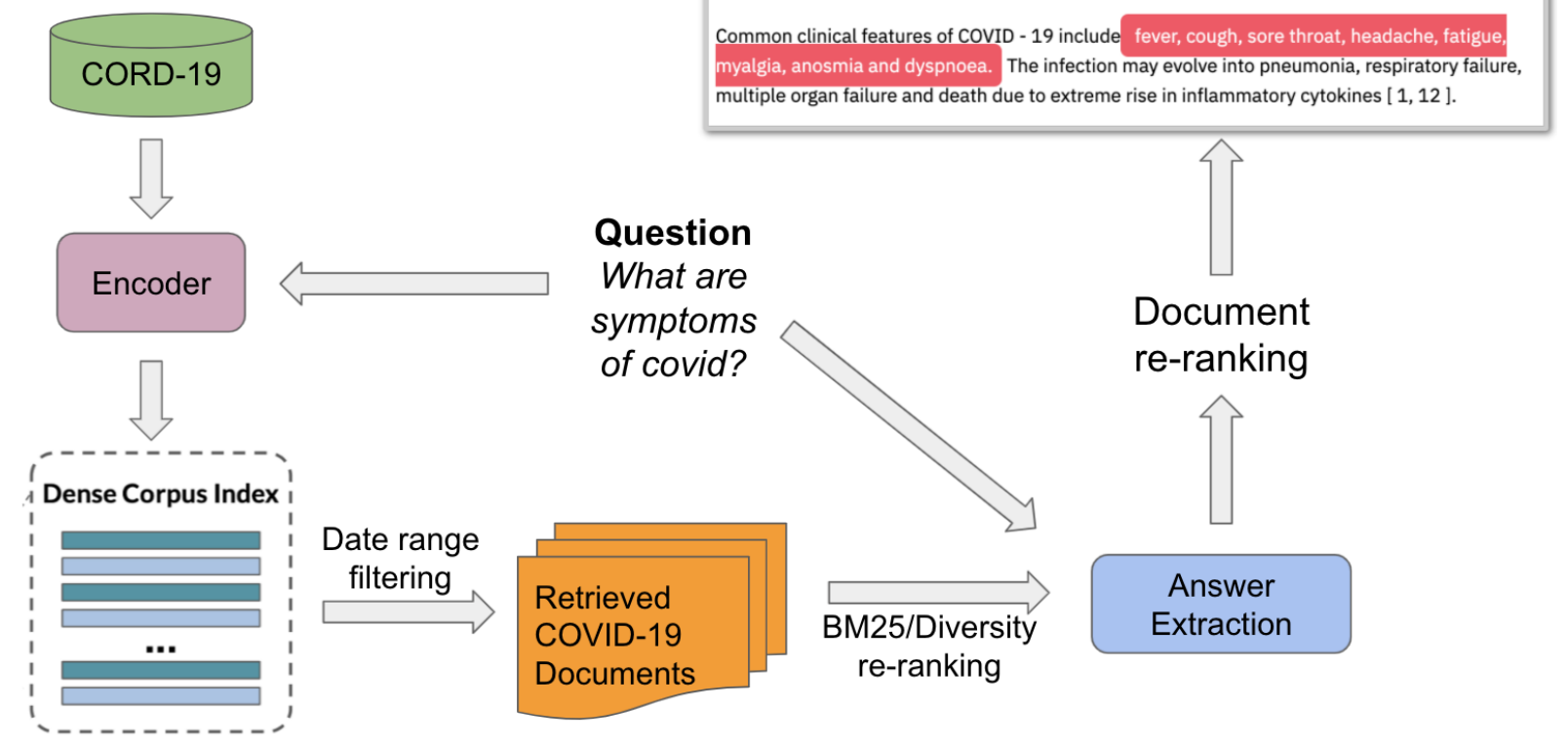

Figure 1: An overview of the COVID-19 open-domain question-answering system. The retrieval component is shown on the left and the reading comprehension/answer extraction component is shown on the right.

19-related question-answering systems have been created in recent months (Bhatia et al., 2020; Yan et al., 2021; Reddy et al., 2020). However, our system incorporates multiple state-of-the-art information retrieval techniques with dense retrieval and BM25 (Robertson and Zaragoza, 2009) and the additional functionality of diversity re-ranking and multiple answer spans.

Our system is comprised of two models: the retrieval model and reading comprehension model. Our system consists of several layers of document and answer re-ranking to increase both quality and diversity in our answers. The overall system can be seen in Figure 1. We additionally provide code ${ }^{2}$ to create an online demo site to visualize our system and provide multiple filters for users to further refine their queries.

Our contributions are

1. We set a precedent for quickly creating an effective open-domain question-answering system for an emergent domain.

2. We integrate multiple stages of document reranking throughout our pipeline to provide relevant and diverse answers.

3. We create an online demo to allow the public

\footnotetext{
${ }^{2}$ https://github.com/sharonlevy/Open_ Domain_COVIDQA
}

to easily obtain answers to COVID-19-related questions from credible scientific sources.

\section{Retrieval}

The retrieval model consists of a dense retriever and contains further layers of re-ranking. In the following sections, we describe the data used to train our model, along with the model details and re-ranking strategies.

\subsection{Data}

As mentioned in Section 1, several COVID-19related datasets have been published throughout the pandemic. However, there are a limited number of sizable datasets focused on the general areas of information retrieval and question-answering. In order to train on in-domain data, we utilize the COVID-QA (Möller et al., 2020) dataset to finetune our model for the document retrieval task. COVID-QA is a COVID-19 question-answering dataset and contains multiple question-answer pairs for each context document (2,019 QA pairs in total), where the documents are COVID-19-related PubMed $^{3}$ articles.

In order to transform the question-answering dataset for our retrieval task, we choose to utilize the questions and their related context articles during training. We split each context article into size

\footnotetext{
${ }^{3}$ https://pubmed.ncbi.nlm.nih.gov/
} 


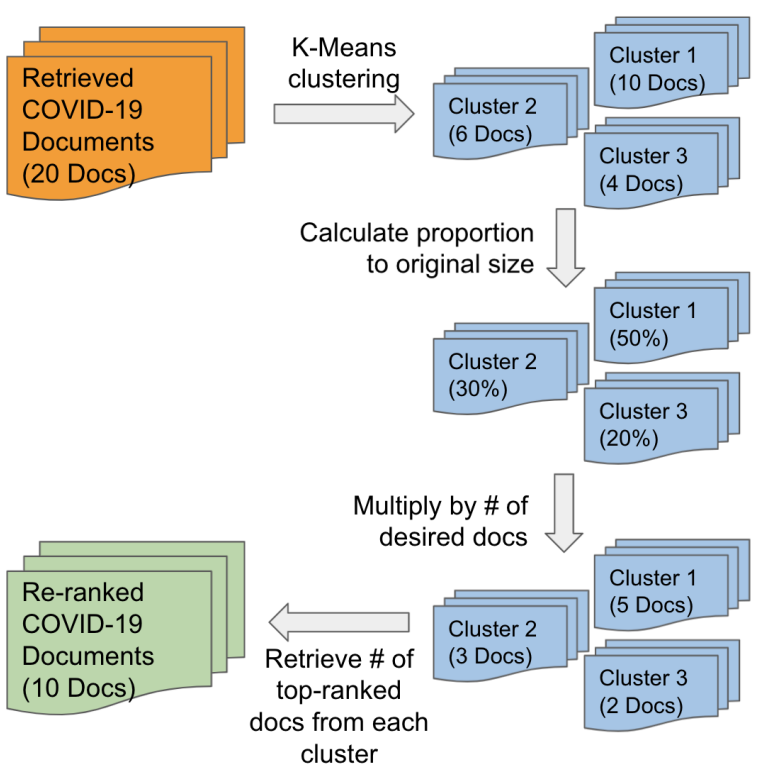

Figure 2: An outline of the diversity re-ranking process discussed in Section 2.4. After the retrieval size for each cluster is calculated, the top-ranking documents (as determined by the hybrid model) are selected from each cluster according to this size and accumulated into the final set of retrieved documents. This final set is also ordered according to the original ranking by the hybrid model.

100-200 tokens. Given the answer for each question and context article pair, we extract only the chunks of text that contain the answer with simple string matching and use this as a positive sample for each question. We further partition the dataset into training, development, and test sets. These splits are made at $70 \%, 10 \%$, and $20 \%$, respectively. Additionally, we remove any document-specific questions (e.g. How many participants are there in this study?) from the test set for a fair assessment.

We utilize the CORD-19 (Wang et al., 2020) dataset as our document corpus for the opendomain retrieval task. The corpus website is consistently updated with newly published COVID-19related papers from several sources. Similar to the COVID-QA dataset, we pre-process each article by splitting it into multiple document entries based on paragraph text cutoffs. Paragraphs that are longer than 200 tokens are split further until they reach the desired 100-200 token size.

\subsection{Dense Retriever}

The dense retriever consists of a unified encoder for encoding both questions and text documents. We utilize the pre-trained PubMedBERT model $(\mathrm{Gu}$ et al., 2020) as the encoder and fine-tune on the

\begin{tabular}{l|c|c|c}
\hline Model & FM@5 & FM@ 20 & FM@ 50 \\
\hline Dense Retrieval & 0.300 & 0.471 & 0.556 \\
\hline BM25 & 0.346 & 0.486 & 0.556 \\
\hline Hybrid Model & $\mathbf{0 . 3 6 2}$ & $\mathbf{0 . 4 9 8}$ & $\mathbf{0 . 6 0 7}$ \\
\hline
\end{tabular}

Table 1: Comparison of dense retriever, BM25, and hybrid models for open-domain retrieval on the test set of COVID-QA. Results are evaluated with fuzzy matching (FM) scores at various retrieval count thresholds. The fuzzy matching process is described in Section 2.5.

COVID-QA dataset. We utilize both positive and negative samples during training. Positive samples consist of paragraphs that contain the exact answer span for the current question. Likewise, negative samples consist of paragraphs that do not contain the exact answer.

During training, the model learns to encode questions and positive paragraphs into similar vectors such that positive paragraphs are ranked higher than negative paragraphs in similarity. After training, the CORD-19 document corpus is passed through the trained encoder and the embeddings are indexed and saved. During test time, the question is used as input to the model. The resulting embedding is used to find similarly embedded documents from the existing dense document embeddings using inner product similarity scores.

\subsection{BM25 Re-ranking}

While the dense retriever excels in the retrieval of documents with semantic similarity to a query, there may be specific keywords in the query that are important for document retrieval. This is especially true in biomedical domains, such as COVID-19, which heavily rely on particular terminology. As a result, our system includes a second stage during retrieval in which we re-rank the top- $n$ retrieved documents with the BM 25 algorithm. Specifically, we use the BM25+ algorithm defined in (Lv and Zhai, 2011). BM25 depends on keyword matching and ranks documents based on the appearance of query terms within the document corpus. We further simplify this by first removing stop words from the top- $n$ documents before re-ranking. We define the combination of our dense retriever with BM25 re-ranking as our hybrid model.

\subsection{Retrieval Diversity}

Following the re-ranking of retrieved documents with BM25, we aim to increase the diversity of 


\begin{tabular}{l|l|c|c}
\hline Model & Datasets & Exact Match & F1 \\
\hline BERT & COVID-QA & 12.27 & 39.07 \\
\hline BERT & SQUAD2.0 & 29.24 & 59.34 \\
\hline BioBERT & SQUAD2.0 & 30.54 & 59.39 \\
\hline BERT & SQUAD2.0 + COVID-QA & 33.68 & 65.53 \\
\hline BioBERT & SQUAD2.0 + COVID-QA & 37.59 & 66.67 \\
\hline BioBERT w/ multiple answer spans & SQUAD2.0 + COVID-QA & $\mathbf{3 9 . 1 6}$ & $\mathbf{7 2 . 0 3}$ \\
\hline
\end{tabular}

Table 2: Comparison of BERT and BioBERT models fine-tuned on combinations of COVID-QA and SQuAD2.0. The final row includes the BioBERT model with multiple answer spans extracted. Each model was evaluated on a held-out test set from COVID-QA.

these documents so that a user does not view nearly identical texts. To do this, we cluster the top- $k$ reranked documents into three clusters with K-Means clustering (MacQueen et al., 1967) and TF-IDF features. For each cluster, we compute its size in proportion to $k$. This relative size is multiplied by the desired number of documents $l$ (where $l<k$ ) to be retrieved. Given the resulting size for each cluster, the most relevant (top-ranked) documents are chosen in their current ranking order. This procedure is illustrated in Figure 2. Following this method allows us to present the user with more diverse and relevant documents that would otherwise be ranked lower.

\subsection{Retrieval Experiments}

We use the test subset of the COVID-QA dataset to evaluate our retrieval model. However, as COVIDQA is intended for the question-answering task, we cannot accurately evaluate our model by simply calculating the retrieval rank of the correct document. This is due to our specific task of opendomain question-answering, in which we are retrieving from the large CORD-19 corpus instead of the much smaller pool of documents in COVIDQA. As a result, we define a fuzzy matching metric to evaluate the quality of our retrieved documents. This is a combination of deep semantic matching and keyword matching. We have varying combinations and thresholds based on respective conditions, such as differing answer lengths. We evaluate the answer in each QA pair in our COVID-QA test set against each retrieved document.

The deep semantic matching is achieved through the Sentence-BERT model (Reimers and Gurevych, 2019) and F1 score is utilized for keyword matching. Each retrieved document is split into a list of sentences and each sentence is evaluated for three conditions:
1. Cosine similarity score that is greater than or equal to threshold $a$ of the sentence/query pair encoded with Sentence-BERT.

2. Cosine similarity score greater than or equal to threshold $b$, where $b<a$, and $\mathrm{F} 1$ score greater than or equal to threshold $c$.

3. F1 score greater than or equal to threshold $d$, where $d>c$. This is only calculated if the token count of an answer is less than or equal to 3 .

If any of the three conditions are achieved for any sentence within the retrieved document, the document is evaluated as a positive retrieval and containing the answer to the query.

We show the impact of the BM25 re-ranking stage in the hybrid model in Table 1. It can be seen that individually, BM25 and the dense retriever models obtain similar retrieval results. However, the hybrid model of dense retrieval followed by BM25 re-ranking allows the system to obtain more relevant documents for the user.

\section{Reading Comprehension}

The second stage of our system consists of a reading comprehension model that can answer the original query based on the retrieved documents. We describe the training data, model design, and document re-ranking associated with our model in the following sections.

\subsection{Data}

We utilize the COVID-QA dataset to train our model for the reading comprehension task. Unlike the retrieval model, the reading comprehension model utilizes both questions and answers, along with their respective context articles for training. As mentioned in Section 2.1, we partition the 


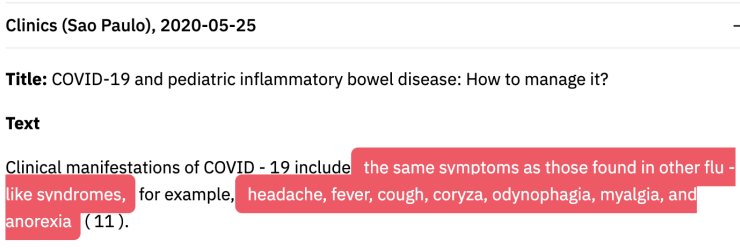

Figure 3: An example of returning multiple answers to a user for the query: "What are symptoms of covid?"

dataset into training, development, and test sets and utilize this to evaluate the model.

\subsection{Methodology}

The reading comprehension model performs extractive question-answering. Given a question and paragraph pair, the model learns to find start and end tokens to represent the answer span (or spans) in the paragraph text. This is done by choosing the highest-ranked start and end tokens produced by the model where the start token is earlier than the end token in the text sequence. We utilize a variant of BioBERT (Lee et al., 2019) that is fine-tuned on the SQuAD2.0 (Rajpurkar et al., 2018) dataset ${ }^{4}$. We find that fine-tuning this model on COVIDQA allows the model to train on both in-domain (COVID-QA) and out-domain (SQuAD2.0) data and increases results for this task when evaluated on the test set of COVID-QA.

\subsection{Multiple Answers}

Some retrieved documents may contain answer spans that are not contiguous. In order to accommodate this, we rank the top- $m$ start and end tokens according to confidence scores and select the pairs of tokens that do not overlap with higher-ranked answer spans. This allows each document to highlight up to $m$ answers rather than just one answer and increases evaluation results. We show the effect of adding multiple answer spans in Table 2 in comparison to various model and fine-tuning dataset combinations. An example of multiple answer spans for a given query can be seen in Figure 3.

\subsection{Document Re-ranking}

When the reading comprehension model is utilized in the overall system, it is used to answer the same question within a set of documents retrieved from the hybrid retriever model. While the documents

\footnotetext{
${ }^{4}$ https://huggingface.co/ktrapeznikov/ biobert_v1.1_pubmed_squad_v2
}

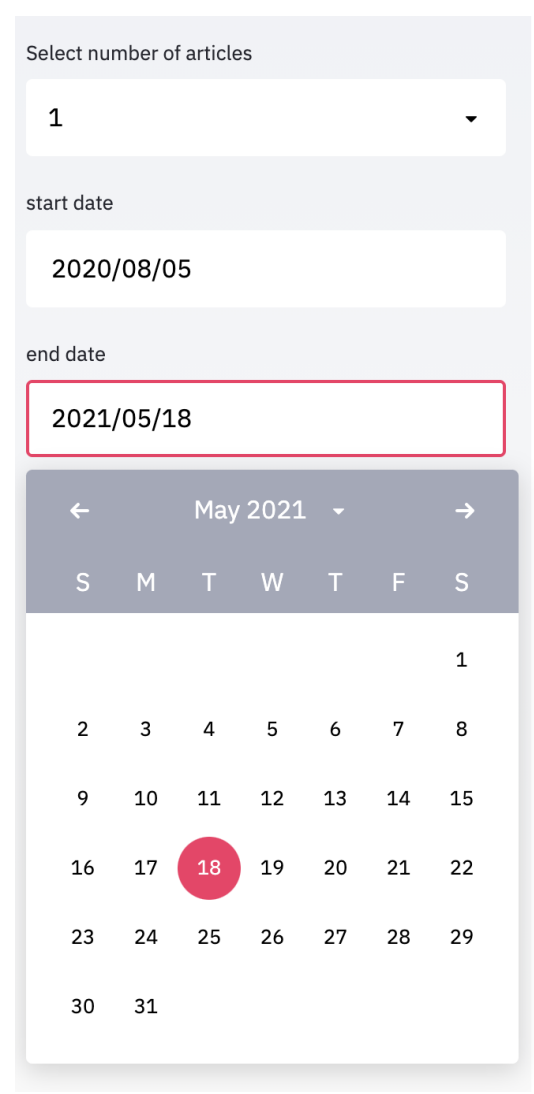

Figure 4: The side panel in the demo website which allows users to filter the number of documents retrieved and the date range for the publication date of these documents.

are already re-ranked by the retriever, we further rerank these documents again following the answer extraction portion of the system. When answering a question for each document, the reading comprehension model provides a confidence score alongside each start and end token. We utilize these confidence scores and reorder the current set of retrieved documents based on the combination of the start and end scores for the top answer in each document. As a result, if a question is not easily answered in a highly ranked retrieved document, the respective document will subsequently be moved to a lower rank.

\section{Open-domain Question Answering}

In the previous sections, we describe the retrieval and reading comprehension models. We combine the two models for the end-to-end open-domain question-answering task. The full system overview can be seen in Figure 1. Once the retriever is trained, the CORD-19 corpus is encoded and stored. When a user queries the system with a question, this question is encoded using the unified retriever 
model and the resulting vector is used to retrieve similar documents from the dense corpus. Once the top documents are retrieved, they are re-ranked with the BM25 algorithm and further clustered/reranked to introduce diversity to the results. The top remaining documents are used as input to the reading comprehension model along with the initial question. This model computes the answer span (and potentially spans) for each document. The documents are then re-ranked given the reading comprehension model's confidence score in the top answer span and the answers for each document are highlighted.

\section{Demo}

We build an online demo that allows users to easily utilize our system. This website is powered through Streamlit ${ }^{5}$.

\subsection{Query Filters}

The input documents for the demo are from the CORD-19 corpus. These documents are preencoded by the trained hybrid retrieval model. We include several features for users to filter in order to narrow down their search. A user is able to decide how many documents they would like to be retrieved (in the range from 1 to 5) from the drop-down menu. We include start and end date selection boxes to allow users to further filter the retrieved documents by publication date within the top retrieved documents. These components are shown in Figure 4. If there are no documents available for the date range, we show this as a message and instead retrieve relevant documents from any date range for the user.

\subsection{Demo Procedure}

The user can enter a free-text question in English into the search bar as seen in Figure 5. This question is encoded by the trained retrieval model and used to find matching documents. The reading comprehension model uses the retrieved documents and query to extract the answer (or answers) and re-rank the documents based on the answer confidence scores. The chosen number of retrieved documents is displayed to the user. Each document is displayed alongside its journal or source name and publication date from its respective CORD-19 article. The user can expand each document heading to view the article title and text snippet. The

\footnotetext{
${ }^{5}$ https://streamlit.io/
}

\section{Ask any question about COVID-19!}

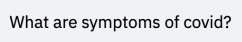

Figure 5: The list of documents returned to a user for a given query. Each document is labeled by its publishing journal and publication date.

\section{Ask any question about COVID-19!}

What are symptoms of covid?

Top 5 Retrieved Articles

Am J Otolaryngol, 2020-08-11

Title: Increased incidence of otitis externa in covid-19 patients

Text

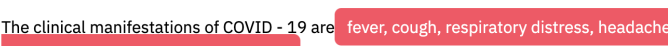

atigue, sore throat, rhinorrhea and GIT symptoms [ 3 ].

Acta Neurol Belg, 2020-06-19

Title: Neurological manifestations of COVID-19, SARS and MERS

Text

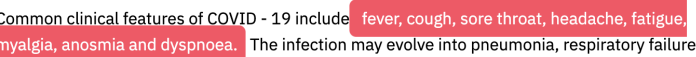
myalgia, anosmia and dyspnoea. The infection may evolve into pneumonia, respiratory fa

Figure 6: Retrieved documents for a given query can be expanded to show their respective article titles and text snippets. Extracted answers for each document are highlighted in red.

extracted answers are highlighted in red as seen in Figure 6.

\section{Conclusion}

In this paper, we present an open-domain question answering system for the emergent domain of COVID-19. Our system is comprised of retrieval and reading comprehension components, with several layers of refinement to increase the quality and diversity of responses. The system allows users to quickly search COVID-19-related questions and obtain a set of answers from biomedical publications. Additionally, we provide a demo website that allows users to easily interact with our system and apply additional filters to further refine their search. We hope that amidst the time of a global pandemic, our system can serve as both a resource 
in finding credible answers to users' COVID-19 questions and a model for future systems in similar emergent domains.

\section{References}

Hunt Allcott and Matthew Gentzkow. 2017. Social media and fake news in the 2016 election. Journal of economic perspectives, 31(2):211-36.

Parminder Bhatia, Kristjan Arumae, Nima Pourdamghani, Suyog Deshpande, Ben Snively, Mona Mona, Colby Wise, George Price, Shyam Ramaswamy, and T. Kass-Hout. 2020. Aws cord19search: A scientific literature search engine for covid-19. ArXiv, abs/2007.09186.

Aengus Bridgman, Eric Merkley, Peter John Loewen, Taylor Owen, Derek Ruths, Lisa Teichmann, and Oleg Zhilin. 2020. The causes and consequences of covid-19 misperceptions: Understanding the role of news and social media. Harvard Kennedy School Misinformation Review, 1(3).

Danqi Chen, Adam Fisch, Jason Weston, and Antoine Bordes. 2017. Reading Wikipedia to answer opendomain questions. In Proceedings of the 55th Annual Meeting of the Association for Computational Linguistics (Volume 1: Long Papers), pages 1870 1879, Vancouver, Canada. Association for Computational Linguistics.

Holly Else. 2020. How a torrent of covid science changed research publishing-in seven charts. $\mathrm{Na}$ ture, pages 553-553.

Yu Gu, Robert Tinn, Hao Cheng, Michael Lucas, Naoto Usuyama, Xiaodong Liu, Tristan Naumann, Jianfeng Gao, and Hoifung Poon. 2020. Domainspecific language model pretraining for biomedical natural language processing. arXiv preprint arXiv:2007.15779.

Mandar Joshi, Eunsol Choi, Daniel S Weld, and Luke Zettlemoyer. 2017. Triviaqa: A large scale distantly supervised challenge dataset for reading comprehension. In Proceedings of the 55th Annual Meeting of the Association for Computational Linguistics (Volume 1: Long Papers), pages 1601-1611.

Ramez Kouzy, Joseph Abi Jaoude, Afif Kraitem, Molly B El Alam, Basil Karam, Elio Adib, Jabra Zarka, Cindy Traboulsi, Elie W Akl, and Khalil Baddour. 2020. Coronavirus goes viral: quantifying the covid-19 misinformation epidemic on twitter. Cureus, 12(3).

Jinhyuk Lee, Wonjin Yoon, Sungdong Kim, Donghyeon Kim, Sunkyu Kim, Chan Ho So, and Jaewoo Kang. 2019. BioBERT: a pretrained biomedical language representation model for biomedical text mining. Bioinformatics, 36(4):1234-1240.
Yuanhua Lv and ChengXiang Zhai. 2011. Lowerbounding term frequency normalization. In Proceedings of the 20th ACM International Conference on Information and Knowledge Management, CIKM '11, page 7-16, New York, NY, USA. Association for Computing Machinery.

James MacQueen et al. 1967. Some methods for classification and analysis of multivariate observations. In Proceedings of the fifth Berkeley symposium on mathematical statistics and probability, volume 1, pages 281-297. Oakland, CA, USA.

Juan Carlos Medina Serrano, Orestis Papakyriakopoulos, and Simon Hegelich. 2020. NLP-based feature extraction for the detection of COVID-19 misinformation videos on YouTube. In Proceedings of the 1st Workshop on NLP for COVID-19 at ACL 2020, Online. Association for Computational Linguistics.

Timo Möller, Anthony Reina, Raghavan Jayakumar, and Malte Pietsch. 2020. COVID-QA: A question answering dataset for COVID-19. In Proceedings of the 1st Workshop on NLP for COVID-19 at ACL 2020, Online. Association for Computational Linguistics.

Tri Nguyen, Mir Rosenberg, Xia Song, Jianfeng Gao, Saurabh Tiwary, Rangan Majumder, and Li Deng. 2016. Ms marco: A human generated machine reading comprehension dataset. In CoCo@NIPS.

Pranav Rajpurkar, Robin Jia, and Percy Liang. 2018. Know what you don't know: Unanswerable questions for SQuAD. In Proceedings of the 56th Annual Meeting of the Association for Computational Linguistics (Volume 2: Short Papers), pages 784789, Melbourne, Australia. Association for Computational Linguistics.

Revanth Gangi Reddy, Bhavani Iyer, Md Arafat Sultan, Rong Zhang, Avi Sil, Vittorio Castelli, Radu Florian, and Salim Roukos. 2020. End-to-end qa on covid-19: Domain adaptation with synthetic training. arXiv preprint arXiv:2012.01414.

Nils Reimers and Iryna Gurevych. 2019. SentenceBERT: Sentence embeddings using Siamese BERTnetworks. In Proceedings of the 2019 Conference on Empirical Methods in Natural Language Processing and the 9th International Joint Conference on Natural Language Processing (EMNLP-IJCNLP), pages 3982-3992, Hong Kong, China. Association for Computational Linguistics.

Kirk Roberts, Tasmeer Alam, Steven Bedrick, Dina Demner-Fushman, Kyle Lo, Ian Soboroff, Ellen Voorhees, Lucy Lu Wang, and William R Hersh. 2020. TREC-COVID: rationale and structure of an information retrieval shared task for COVID-19. Journal of the American Medical Informatics Association, 27(9):1431-1436.

Stephen Robertson and Hugo Zaragoza. 2009. The probabilistic relevance framework: BM25 and beyond. Now Publishers Inc. 
Gerard Salton and Michael J McGill. 1986. Introduction to modern information retrieval.

Raphael Tang, Rodrigo Nogueira, Edwin Zhang, Nikhil Gupta, Phuong Cam, Kyunghyun Cho, and Jimmy Lin. 2020. Rapidly bootstrapping a question answering dataset for covid-19. arXiv preprint arXiv:2004.11339.

Samia Tasnim, Md Mahbub Hossain, and Hoimonty Mazumder. 2020. Impact of rumors and misinformation on covid-19 in social media. Journal of preventive medicine and public health, 53(3):171-174.

George Tsatsaronis, Michael Schroeder, Georgios Paliouras, Yannis Almirantis, Ion Androutsopoulos, Eric Gaussier, Patrick Gallinari, Thierry Artieres, Michael R Alvers, Matthias Zschunke, et al. 2012. Bioasq: A challenge on large-scale biomedical semantic indexing and question answering. In 2012 AAAI Fall Symposium Series.

Lucy Lu Wang, Kyle Lo, Yoganand Chandrasekhar, Russell Reas, Jiangjiang Yang, Doug Burdick, Darrin Eide, Kathryn Funk, Yannis Katsis, Rodney Michael Kinney, Yunyao Li, Ziyang Liu, William Merrill, Paul Mooney, Dewey A. Murdick, Devvret Rishi, Jerry Sheehan, Zhihong Shen, Brandon Stilson, Alex D. Wade, Kuansan Wang, Nancy Xin Ru Wang, Christopher Wilhelm, Boya Xie, Douglas M. Raymond, Daniel S. Weld, Oren Etzioni, and Sebastian Kohlmeier. 2020. CORD-19: The COVID-19 open research dataset. In Proceedings of the 1st Workshop on NLP for COVID-19 at ACL 2020, Online. Association for Computational Linguistics.

Wenhan Xiong, Xiang Lorraine Li, Srinivasan Iyer, Jingfei Du, Patrick Lewis, William Yang Wang, Yashar Mehdad, Wen-tau Yih, Sebastian Riedel, Douwe Kiela, and Barlas Oğuz. 2021a. Answering complex open-domain questions with multi-hop dense retrieval. International Conference on Learning Representations.

Wenhan Xiong, Hong Wang, and William Yang Wang. 2021b. Progressively pretrained dense corpus index for open-domain question answering. In Proceedings of the 16th Conference of the European Chapter of the Association for Computational Linguistics: Main Volume, pages 2803-2815, Online. Association for Computational Linguistics.

Rui Yan, Weiheng Liao, Jianwei Cui, Hailei Zhang, Yichuan $\mathrm{Hu}$, and Dongyan Zhao. 2021. Multilingual COVID-QA: Learning towards Global Information Sharing via Web Question Answering in Multiple Languages, page 2590-2600. Association for Computing Machinery, New York, NY, USA.

Wei Yang, Yuqing Xie, Aileen Lin, Xingyu Li, Luchen Tan, Kun Xiong, Ming Li, and Jimmy Lin. 2019. End-to-end open-domain question answering with bertserini. In Proceedings of the 2019 Conference of the North American Chapter of the Association for
Computational Linguistics (Demonstrations), pages 72-77. 